\title{
Steerable3D: an ImageJ plugin for neurovascular enhancement in 3-D segmentation
}

\author{
Paolo Miocchi ${ }^{1}$, Alejandra Sierra ${ }^{2}$, Laura Maugeri ${ }^{1}$, Eleonora Stefanutti ${ }^{1}$, Ali Abdollahzadeh ${ }^{2}$, \\ Fabio Mangini ${ }^{1}$, Marta Moraschi ${ }^{3}$, Inna Bukreeva ${ }^{4}$, Lorenzo Massimi ${ }^{4,5}$, Francesco Brun ${ }^{4,6,}$ \\ Jussi Tohka ${ }^{2}$, Olli Gröhn ${ }^{2}$, Alberto Mittone ${ }^{7,8}$ Alberto Bravin $^{7}$, Federico Giove $^{3}$, Alessia Cedola ${ }^{4}$ \\ \& Michela Fratini ${ }^{1,4}{ }^{*}$
}

${ }^{1}$ IRCCS Fondazione Santa Lucia, Roma, Italy

${ }^{2}$ A.I. Virtanen Institute for Molecular Sciences, University of Eastern Finland, Kuopio, Finland

${ }^{3}$ Centro Fermi - Museo Storico della Fisica e Centro Studi e Ricerche Enrico Fermi, Roma, Italy

${ }^{4}$ Institute of Nanotechnology, Consiglio Nazionale delle Ricerche, Roma, Italy

${ }^{5}$ Department of Engineering and Architecture, University of Trieste, Trieste, Italy

${ }^{6}$ Department of Medical Physics and Biomedical Engineering, University College London, Gower St, London WC1E 6BT, UK

${ }^{7}$ European Synchrotron Radiation Facility (ESRF), Grenoble, France

${ }^{8}$ CELLS ALBA SYNCHROTRON LIGHT SOURCE, Barcelona, Spain

${ }^{*}$ Corresponding author:

E-mail: michela.fratini@gmail.com (MF), paolo.miocchi@gmail.com (PM)

\begin{abstract}
Purpose: Medical image processing plays a fundamental role in the study of central nervous system, with important implications in the analysis of the neural and vascular networks relationships, as well as on pre-clinical investigations about neurodegenerative diseases. Nonetheless, the multi-scale nature of vessels and nerve fibres, the presence of background noise and of strong contrast inhomogeneities, still represent major obstacles to an accurate and computational efficient image
\end{abstract}


segmentation.

In this work we report the implementation of a three-dimensional Gaussian steerable filter tuned up for the enhancement of tubular structures in $3 \mathrm{D}$ images.

Methods: Relevant tests of the filter application are presented, for both synthetic and high resolution Synchrotron X-ray Phase Contrast micro-Tomography ( $\mathrm{SxrPC} \mu \mathrm{T})$ images of an ex-vivo mouse spinal cord (SC), with different sample preparations.

Results: The filter response shows a strong amplification of the source image contrast-to-background ratio (CBR), independently of structures orientation. Such a result provides a great help in the extraction and rendering of entire three-dimensional (3D) neurovascular networks, even in the presence of a noisy background. After the filter application, the CBR ratio increases by a factor ranging from $\sim 6$ to $\sim 60$.

Conclusion: The developed tool (Steerable3D) can generally facilitate the detection/segmentation of tiny blood vessels, connections, neuronal bodies and axonal fibers that were not clearly observable in non-filtered SxrPC $\mu \mathrm{T}$ images. Its systematic application could allow obtaining quantitative information from pre-clinical and clinical images. The Steerable3D tool is made publicly available as an open-source and user-friendly plugin for the ImageJ software platform.

Key words: X ray Phase contrast Tomography, vascular network, 3D steerable filter 


\section{Introduction}

The segmentation of vascular networks (VNs) in the central nervous system (CNS) is of crucial importance for clinical and pre-clinical image processing applications (see, e.g., [1] for a review on this subject). In particular, the three-dimensional (3-D) analysis (determination of crucial parameters such as density, distribution and continuity of vessels) of the VN (including its smallest capillaries), is one of the key issues in neurodegenerative diseases, such as multiple sclerosis [2-4], where pathologic neurovascular alterations have been regarded as a key process $[2,3]$. In this framework, the knowledge of the relative spatial location of blood vessels with respect to neurons and axons is mandatory for improving our understanding of the effects of pathological processes.

To this aim, a 3-D high-resolution micro-imaging technique is required to define the complex structure of the $\mathrm{VN}$ in the central nervous system. Magnetic resonance angiography, volumetric computed tomography [5] and conventional X-ray angiography [6] have been used to image the neural vasculature, but their low resolution is inappropriate for imaging small vessels $(<20 \mu \mathrm{m})$. Highresolution synchrotron tomography has been proposed for the complete $3 \mathrm{D}$ imaging of micro-VNs [6], yet a better contrast is achieved by imaging the phase modulation induced by an object in a coherent beam $[7,8]$. This latter feature makes Synchrotron X-ray Phase-contrast micro-Tomography ( $\mathrm{SXrPC} \mu \mathrm{T})$ a very attractive method to study weakly absorbing samples, such as the spinal cord vascular and neuronal network. In particular, $\mathrm{SXrPC} \mu \mathrm{T}$ provides an excellent 3-D resolution allowing us to detect the arteries, veins, capillaries, neuronal cells and axonal fibers at the micron scale with no contrast agent $[9,10]$.

However, for the phase-contrast tomography data the use of no contrast agents makes vessels difficult to segment using intensity-based image processing approaches, such as choosing an appropriate threshold, because the intensity values of the vessels strongly overlap with those pertaining to other structures and to the background. In addition, the segmentation of VNs is seriously hampered by the presence of image noise and strong contrast inhomogeneities, as well as by the multi-scale nature of vessels and nerve fibres, which prevents from using size based automated recognition algorithms. For 
all these reasons, the development of a computationally efficient 3D segmentation tool for the vascular network is an extremely challenging task.

In this respect, a large variety of methods have been set up, mostly of which relying on the morphological properties of "tubular structures". Very simple vessel detection techniques were developed many years ago (based on global or locally adaptive thresholds developed in a more general context $[11,12])$, and have been extensively used because of their conceptual simplicity and low computational cost. Their main drawback is the need of a careful fine-tuning in parameters selection to avoid inaccurate results. Recently, more sophisticated techniques have been introduced following an iterative approach in which, starting from a set of manually chosen "seed points", the segmentation proceeds propagating towards peripheral branches by means of active contours [13], region growing $[14,15]$ and particle filtering or path tracing [16-19] thus exploiting the interconnected, "graph-like", topological structure of vessels.

However, a requisite building-block in such algorithms is the measure of tubular degree or "vesselness", that is more commonly evaluated by means of Hessian-based methods. These rely on the eigenstate analysis of the Hessian, estimated from the second-order derivatives of local intensity levels, which can then be used to define a vesselness as a function of the eigenvalues [18, 20-22]. Nonetheless, in order to deal with the multi-scale nature of vascular structures, Hessian-based filters usually include a preliminary Gaussian convolution that has to be re-applied each time at the different scales. This tends to blur vessel boundaries, which could make scale selection inaccurate, especially close to bifurcations. To overcome this issue various authors proposed more sophisticated versions of this approach, such as bi-Gaussian pre-filtering [23] and oriented flux calculations at the boundary of localized circles (2-D) or spheres (3-D) with different radii (i.e. scales) [24, 25]. Unfortunately, these improvements imply a non-negligible computational extra-cost to pay for.

Alternative techniques to enhance tubular structures include optimal edge detection [11] and steerable filters [26, 27]. Among these techniques, we chose to implement a Gaussian steerable filter [28] because it allows to achieve a good compromise between computational cost and features contrast 
enhancement. Indeed, this filter shows two important properties: i) it has an explicit scale parameter that allows us to optimize the filter response (FR) to take into account the multi-scale nature of vascular structures;and ii) the filter convolution can be computed very efficiently for any arbitrary orientations thanks to the steerability property of Gaussian derivatives [18]

In this paper we describe an implementation of this filter within the ImageJ integrated graphical platform (https://imagej.net). ImageJ is a public domain and open-source image processing software tool whose main strengths are modularity, portability and computational efficiency [28]. For these reasons, we decided to implement it as a (publicly available and open-source) plugin that can be integrated in the platform. To our knowledge no 3-D steerable filters are available as a plugin for ImageJ (see [27] for a 2-D implementation). Finally, we report on various filter quality tests applied to $\mathrm{SXrPC} \mu \mathrm{T}$ mouse spinal cord images.

\section{Materials and methods}

\section{Sample preparations}

We studied the spinal cord of healthy adult male C57BL/6J mice (20-22 g, body weight).

We measured 12 spinal cord samples from ex-vivo adult male C57BL/6J mice(5-6 weeks old, weight 21-28 g, Charles River). The animals were housed in a room $\left(22 \pm 1{ }^{\circ} \mathrm{C}, 50 \%-60 \%\right.$ humidity $)$ with 12 $\mathrm{h}$ light/dark cycle and free access to food and water. The samples were divided in four groups. One group was filled with physiological solution; another was perfused with MICROFIL ${ }^{\circledR}$, a low-viscosity radio-opaque polymer (Flowtech, Inc., Carver, Massachusetts) well suited for vascularization studies; a third group was perfused with ethanol and the last one was perfused with paraformaldehyde (PFA). The detailed sample preparation protocol is reported in [29]. These experimental animal procedures was be carried out at the A.I.Virtanen Institute for Molecular Sciences at the University of Eastern Finland (Kuopio, Finland) and approved by the Animal Care and Use Committee of the Provincial Government of Southern Finland and performed according to the guidelines set by the European Community Council Directive 86/609/EEC. 


\section{$\mathrm{SXrPC \mu T}$ measurements}

In-line SXrPC $\mu \mathrm{T}$ measurements were carried out at the I13-2 beamline of Diamond Light Source (Harwell Science and Innovation Campus, Oxfordshire, UK) and at the ID17 beamline of ESRF

(Grenoble, France). At I13-2 tomographic images were collected using a pseudo-monochromatic $\mathrm{X}$-ray beam with main peak at $14.7 \mathrm{keV}$ and a system of magnification optics connected to a PCO.Edge 5.5 camera to get an affective pixel size of about $1.625 \mu \mathrm{m}$; the sample was located $70 \mathrm{~cm}$ far from the detector. At ID17 a monochromatic X-ray beam at the energy of $30 \mathrm{keV}$ was used, in combination with a sample-detector distance of $2.0 \mathrm{~m}$ and a detection pixel size of $3.5 \mu \mathrm{m}$ [30].

Phase retrieval was performed applying the single distance method proposed by Paganin [31] and all projections were processed and reconstructed using an open-source software tool SYRMEP Tomo Project [32]. We thus obtained a set of high-spatial resolution tomographic images, where the different grey levels are proportional to the electron density of the different tissues inside the sample.[7, 8]. The reconstructed volumetric images were analysed and filtered by means of ImageJ, using the homedeveloped plugin as described below.

For a proper comparison of the original volumetric images with the filtered ones we made a 2-D projection of their voxels. Let $x$ and $y$ be the horizontal and vertical axes, respectively, on the spinal cord axial plane, so that the $z$-axis lies along the images stack axis (i.e. perpendicular to the axial plane). Each non-filtered volume of interest (VOI) was thus projected through the following steps:

1) taking the "projected minimum" of the VOI. This was done by creating a 2-D image in which the grey level $p$ of the generic pixel at $(x, y)$ is given by

$$
p(x, y)=\min _{0 \leq z \leq Z}\{v(x, y, z)\}
$$

where $v(x, y, z)$ is the grey level of the VOI voxel at $(x, y, z)$, with $Z$ being the upper limit of the $z$ coordinate (it coincides with the number of slices in the stack).

2) Taking the "projected maximum" of the VOI (i.e. applying Eq eq_1, but with the "max" instead of the "min" operator).

3) Summing (pixel-by-pixel) the image of step (1) (after inverting their grey levels) with the 
projected maximum of step (2).

For grey scale "inversion" we mean the transformation of the grey level $p$ of a pixel to $M-p+m$, with $m$ and $M$ being, respectively, the minimum and maximum grey level of the image (notice that the absolute value of the difference between the grey levels of any pixels pair is invariant under this transformation).

Indeed, the projected minimum and maximum are commonly used to segment projected neurovascular network in images stack (see, e.g. ref. $[2,9]$ ) because, on the one hand, the resulting dark pixels very likely come from vessels lumen and, on the other hand, cell bodies tend to give rise to bright pixels. Moreover, as we will see below, since in the filtered image the vessels' pixels are brighter than the background, we transformed the dark vessels of the non-filtered image into bright objects by inverting the projected minimum, so to make the image more easily and visually comparable with the projected FR. The latter was obtained by doing only the "projected maximum" on the 3-D FR voxels. Indeed, the grey level in the FR can be regarded as a "vesselness" measure.

\section{The "Steerable3D" tool}

The 3-D segmentation of the VN, up to the capillary network (on $\sim 5 \mu \mathrm{m}$ of length-scale), makes the adoption of a computationally efficient filter in 3-D of crucial importance for medical application. For this reason, we implemented a filter based on 3-D Gaussian derivatives which allows to filter along any arbitrary orientation with a small computational extra-cost. The result of the filtering procedure - the FR - is obtained by the convolution of the image with the filter template (FT, see Fig. 1). Specifically, the convolution consists in a weighted average of all the image pixels around a given one, with the weights given by the FT (see below). Moreover, the FT can be easily re-scaled so as to enhance vessels/fibres of different size (from $\sim 1$ to $\sim 30 \mu \mathrm{m}$ ).

The filter implementation was done as a form of an ImageJ (open-source) plugin that we called "Steerable3D" (S3D). It is based on the theoretical approach described by Schneider and colleagues 
[27] in which the adopted FTs are written as normalized derivatives of Gaussians, that is:

$$
G_{M, a, b}^{\sigma}(\vec{x})=\sigma^{M} \frac{\partial^{M-a} \partial^{a-b} \partial^{b}}{\partial_{x}^{M-a} \partial_{y}^{a-b} \partial_{z}^{b}} G^{\sigma}(|\vec{x}|)
$$

where the triplet $(M, a, b)$, with $M>0,0 \leq a \leq M$ and $0 \leq b \leq a$, identifies the template "order" and $G^{\sigma}(x)$ is the gaussian with variance $\sigma^{2}$ and zero mean. In Fig. 1, the form of the FT at various orders is illustrated.

In addition to having an explicit and easily adjustable scale parameter $(\sigma)$, this filter is "steerable" also in 3-D (as was demonstrated in [28], see also [26, 33]). Steerability is the property whereby the convolution of an image with a rotated version of the FT can be expressed as a linear combination of various orders of the same FT without rotation (Fig. 1). In formal notation, given the FR, i.e. the convolution of the image $I$ with the FT at the voxel $\vec{x}(x, y, z)$, as

$$
f_{M, a, b}^{\sigma}(I, \vec{x})=\left(I * G_{M, a, b}^{\sigma}\right)(\vec{x}), \quad \text { (eq_3) }
$$

then the convolution with the FT rotated in 3-D with a given elevation $(\theta)$ and azimuth $(\varphi)$ angles (with respect to the axial, slice, $x y$ plane, see Fig 2) can be written as

$$
f_{M, a, b}^{\sigma}(I, R \vec{x})=\sum_{i=0}^{M} \sum_{j=0}^{i} w_{M, a, b}^{i j}(\theta, \varphi) f_{M, i, j}^{\sigma}(I, \vec{x})
$$

where $R=R(\theta, \varphi)$ is the corresponding rotation matrix and $w^{i j}, M a, b(\theta, \varphi)$ is a set of coefficients depending on the angles (Ref. [28], Sect. 2.1.2, for further details). As a consequence, for the sake of computational convenience and rapidity, the evaluation of the rotated FR can be done by using Eq. (eq_4) instead of the direct and much more computational expensive convolution (eq_3) re-evaluated at $R \vec{x}$. In the plugin, the optimal orientation is found for each voxel as that giving the maximum FR among the responses corresponding to the angles:

$$
\begin{gathered}
\theta=\theta_{1}, \theta_{1}+\Delta \theta, \theta_{1}+2 \Delta \theta, \ldots, \theta_{2} \\
\varphi=\varphi_{1}, \varphi_{1}+\Delta \varphi, \varphi_{1}+2 \Delta \varphi, \ldots, \varphi_{2}
\end{gathered}
$$

where the angular bounds $0 \leq \theta_{1}<\theta_{2} \leq 90^{\circ}, 0 \leq \varphi_{1 \square}<\varphi_{2} \square \leq 360^{\circ}$, and the steps $\Delta \theta, \Delta \varphi$ can be given by the user (see next Section). Notice that only one half-space (with $z \geq 0$ ) needs to be considered because all the FTs are invariant under the $\theta \rightarrow-\theta$ transformation. 


\section{Results}

\section{The S3D workflow}

The S3D plugin is written in Java and it is capable to run in parallel on multi-CPU computers thanks to the native multithreading mechanism of Java language. Since each thread can operate independently of each other on a subset of all the slices composing the 3-D stack, the computational parallelization can be done straightforwardly in both the calculation stages and the performance shows a good scalability (see Fig. 3 for a sketch of the tool workflow).

S3D operates on 32-bit (single precision floating point) greyscale images, and therefore other types of images must be converted before being treated (ImageJ has an embedded conversion tool that is accessible through the Image -> Type menu path). Moreover, it needs 3-D images, thus a stack of 2-D image slices must be loaded first. Then, the plugin can be launched and a GUI opens (Fig. 4). The user can first select whether to apply a "custom" FT order by giving the values for $M, a$ and $b$, or to set a pre-defined FT. S3D provides three different pre-defined templates, namely: i) the "Edge" type that corresponds to $(M, a, b)=(1,0,0)$ and yields an edge detector, ii) the "Ridge" type, with order $(2,1,0)$, that gives a high response when a linear bright series of voxels is surrounded by darker voxels and iii) the order $(2,0,0)$ that we name "Vessel" type because it yields a high response when a darker set of aligned voxels is confined within brighter "boundaries" (see Fig 1). The user can then set the "scale" parameter (corresponding to $\sigma$ in Eq eq 2 ), as a length-scale parameter for the FT, as well as the angular limits and the steps used in the search for the optimal filter orientation (Fig. 4). When the "Run" button is pressed the plugin starts to calculate the filter convolution (without rotation, Eq eq_3) with the whole image. The progression of this stage is shown in the progress bar of the ImageJ main window (Fig. 3), along with the information on how many threads are being used in this calculation. After that, the searching for the optimal rotation angle is executed (applying Eq eq_4) while the progress bar and the number of threads are displayed also for this stage. At the end a new image stack is displayed containing the 3-D FR. Finally, notice that if the button "Draw template" is 
pressed, then the plugin will just display the chosen FT form; therefore only the filter order is taken into account in this case.

\section{Steerable3D testing and application}

As a first step, we applied S3D to simple synthetic images. In Fig. 5 B we can see a 3-D view of synthetic "vessels" represented by cylinders immersed in additive white noise (in Fig. 5 A the "vascular network" is reported without noise for illustrative purposes). The size of vessels was either 2 or 8 voxels. In Fig. 5 C,D,E the FRs are reported for the "Vessel" FT (order 2,0,0) with scale parameter $\sigma=2,4$ and 8 , respectively. We can see that the filter behaves as expected as it enhances the contrast between vessels walls and the (noisy) background. It is also apparent that the larger the scale the lower the noise level in the response but the greater the accuracy of vessel discrimination. To this respect, in Fig. $5 \mathrm{E}$ it is worth noting that the cylinders appear dilated.

As a realistic test of S3D, we examined $\mathrm{SXrPC} \mu \mathrm{T}$ images. For the easiest comparison of the original volumetric images with the filtered ones, we made a 2-D projection of their voxels. Specifically, we took the "projected minimum" and the "projected maximum" of the VOI from the non-filtered image stack (as commonly done to segment projected VN [2,9]). Then, we summed, pixel-by-pixel, the inverted grey levels of the former with those of the latter, where the inverted grey levels were calculated as previously described (ref. to sec. "SXrPC $\mu \mathrm{T}$ measurements) in order to turn dark pixels (which mainly correspond to vessel lumens) into bright ones. Finally, only the projected maximum was applied to the filtered VOI.

In Fig. 6 A, we selected a small VOI in the central part of a stack of 100 greyscale slices of a mouse spinal cord injected with MICROFIL ${ }^{\circledR}$ (this sample was measured at ID17 with a pixel size of about 3.5 micron)

In Figs. $6 \mathrm{~B}, \mathrm{C}$ we can see how in the S3D response of the VOI the blood vessels contrast is clearly enhanced against the background pixels, revealing more details and vascular network connections. The non-filtered projected image was obtained through the above-described procedures, while the FR 
projected in Fig. $6 \mathrm{C}$ was obtained by doing only the "projected maximum" on the 3-D FR voxels. The image in Fig. $6 \mathrm{C}$ was rendered in red and, finally, in Fig. 7 the FR is rendered in 3-D to appreciate the filtered vessels global structure.

In Fig. 8 the same comparison between non-filtered and filtered images was done in the same spinal cord region, on samples without contrast agent but only perfused with PFA or ethanol (these samples were measured at I13 with a pixel size of 1.6 micron) used as fixative agents [29]. Also in this case the filter greatly improves the general visibility of both blood vessels and neural fibres in the white matter region (we notice how the nerve fibres visibility is especially enhanced in the ethanol perfused specimen, Figs. 8 C,D).

The enhancement of the neurovascular system components that is apparent in the above-reported SxrPC $\mu \mathrm{T}$ images, can be rigorously quantified by measuring the contrast-to-background ratio (CBR, see. e.g., Ref. [34]). This can be defined as

$$
c_{\mathrm{X}}=\frac{s_{\mathrm{X}}-B_{\mathrm{X}}}{\left|B_{\mathrm{X}}\right|}
$$

where the ' $\mathrm{X}$ ' index represents the component - or detail - we are interested in, $S_{\mathrm{X}}$ is the maximum grey level in the set of pixels within a region enclosing a significant part of that component and of the adjacent area (see, e.g., Fig. 8), and $\mathrm{B}_{\mathrm{X}}$ is the background level, determined by taking the minimum grey level within the same region in which $S_{\mathrm{X}}$ was taken (of course $S_{\mathrm{X}} \geq B_{\mathrm{X}}$ by definition). In the following table $1, \mathrm{X}={ }^{\prime} \mathrm{N}$ ' stands for neural cell bodies, ' $\mathrm{V}$ ' for blood vessels and 'I' for the whole image.

We evaluated $c_{\mathrm{X}}$ both in the non-filtered image and in the filter response $(c \mathrm{X}, \mathrm{FR})$, thus obtaining a sort of 'filter gain' defined as the ratio $c_{\mathrm{X}, \mathrm{FR}} / c_{\mathrm{X}}$. This gain measures the "efficiency" of the filter application, i.e. how many times it increases the CBR of the relevant details with respect to their surroundings. Of course, care was taken to ensure that the same component and the same region were considered in both the filtered and non-filtered image to evaluate $S_{\mathrm{X}}$ and $B_{\mathrm{X}}$. Resulting values are reported in Table 1.

Another possible application of this filter is to provide support for grey matter (GM) segmentation in 
spinal cords. Indeed, the differences in the vascular network structure between GM and white matter, yields a substantially different response, as we can see in Fig. 9. It is evident that the GM is globally more clearly distinguishable in the projected maximum of the FR (Fig. 9 B) than in the projected maximum (Fig. 9 A) of the original non-filtered stack. In particular, the FR gives rise to a rather sharp region with a peculiar pixels "texture" corresponding to the GM region, which can thus be more easily segmented.

\section{Discussion}

In this work the implementation of "Steerable3D", a 3-D steerable Gaussian filter for the visual enhancement of multi-scale neurovascular networks in $3 \mathrm{D}$, is described and tested. The implementation is done in form of a publicly available, open-source and user-friendly plugin for the ImageJ modular software platform [35]. We preliminarily checked the filter response on a synthetic "vascular" image (represented by tubular structures) and we verified that S3D is able to extract and enhance the tubular features from the background. Moreover, we tested S3D on high-resolution SxrPC $\mu \mathrm{T}$ images of ex-vivo mouse spinal cord to show the performances of the filter under realistic conditions (differing in sample preparation, X-ray source and resolution). The tests confirm that S3D is able to significantly enhance the visibility of blood vessels, nerve fibres and neuronal cell bodies against the background voxels. This enhancement is confirmed by the comparison of the CBR (Eq [eq_6]) measured before and after the filter application.

Values found for this ratio are listed in Table 1, where we can see that for blood vessels S3D can amplify the CBR ratio by a factor that ranges from $\sim 6$ to $\sim 60$, depending on the sample preparation/condition.

The above mentioned CBR can be visually and qualitatively understood also by observing the distribution of grey levels in the image pixels, reported in Fig. 10. The surface plot depicted in the figure refers to PFA-perfused case images. On the $z$-axis the grey level of every pixel in $(x, y)$ of the images in Figs. 8 A,B is reported. We can immediately see that the "ranges of peaks" are higher and 
sharper in the filtered image than in the non-filtered one, with respect to the surrounding "valleys" of the surface plot. The lower grey levels associated to neurons in the FR in this case can be explained by the relatively high resolution of the image that allows to better reproduce the "irregular" ("starred") form of their soma, thus generating lower vesselness values in the FR.

\section{Conclusion}

In summary and conclusion, the "Steerable3D" (S3D) filter, was developed to enhance the visibility of multi-scale neurovascular networks in three-dimensional images. The tests, carried out on highresolution SxrPC $\mu \mathrm{T}$ images of ex-vivo mouse spinal cord, demonstrated that S3D can generally facilitate the detection/segmentation of smallest blood vessels, connections, neuronal bodies and axonal bundles that were not clearly observable in the non-filtered images.

It is worth mentioning that in its present version, S3D does not exploit the information about the template orientation that the employed algorithm uses internally to search for the best local response. In a forthcoming version of the tool, we plan to use this information with a twofold aim: i) as a guide for a skeletonization procedure (the optimal angles naturally indicate the main vessel/fibre local orientation), ii) to help discriminating neuronal cell bodies from vessels and nerve fibres. Indeed, as illustrated in Fig. 8, also these cell bodies are enhanced by the filter. This is because neurons have a roughly ellipsoidal geometrical structure that resembles (on a certain scale) that of a small segment of a tubular structure. On the other hand, the distribution of the optimal filter orientation on the cell body surface is different from that on a real vessel segment. In particular, it is expected that the average of the unit vectors corresponding to orientations of detected structures should be substantially different in the two cases. Therefore, in the future we plan to use this feature to mask the filter response in such a way to discriminate vessel/fibres from neurons.

Another promising line of application is the use of S3D as a 'global' segmentation tool for white/grey matter in SC. Indeed, the very different nerve fibres and blood vessels density in the two regions lead to a rather different filter global response, which appear as regions with a very different graphical 
"texture" (see Fig. 9), thus allowing to distinguish between each other much more easily than in the original non-filtered image.

Finally, we remark the importance of having developed an open-source tool that can be easily extended/updated - also by contributors coming from the scientific community - in order to improve selectivity for the segmentation of the relevant features and to increase computational efficiency.

\section{Acknowledgements}

Authors wish to thank the I13-2 and ID17 beamlines, at the Diamond Light Source and ESRF, respectively, for providing beamtime. They are also grateful to both the beamline staffs for their helpful scientific and technical support. In addition, they would like to thank Maarit Pulkkinen for her help with the animal handling. Finally, P. Miocchi warmly thanks Simone Melchionna and Marco Montuori for the helpful discussions on image processing algorithms.

Funding: This work was supported by the Italian Ministry [Young Researcher Grant 2013, GR-201302358177] and by European projects MSCA RISE 69110 MICROBRADAM and VOXEL (HORIZON 2020-Fet Open; 665207). A. Sierra also acknowledges the Academy of Finland (\#275453) for financial support A. Bravin acknowledges COST Action CA16122( BIONECA) and CNR Italy for financial support. . 


\section{References}

[1] Lesage D, Angelini ED, Bloch I, Funka-Lea G. A review of 3D vessel lumen segmentation techniques: Models, features and extraction schemes. Medical Image Analysis. 2009;13:819-45.

[2] Cedola A, Bravin A, Bukreeva I, Fratini M, Pacureanu A, Mittone A, et al. X-Ray Phase Contrast Tomography Reveals Early Vascular Alterations and Neuronal Loss in a Multiple Sclerosis Model. Scientific Reports. 2017;7:5890.

[3] MacMillan CJ, Starkey RJ, Easton AS. Angiogenesis is Regulated by Angiopoietins During Experimental Autoimmune Encephalomyelitis and is Indirectly Related to Vascular Permeability. Journal of Neuropathology \& Experimental Neurology. 2011;70:1107-23.

[4] Massimi L, Fratini M, Bukreeva I, Brun F, Mittone A, Campi G, et al. Characterization of mouse spinal cord vascular network by means of synchrotron radiation X-ray phase contrast tomography. Physica Medica. 2016;32:1779-84.

[5] Siegel A SH. 2011.

[6] Risser L, Plouraboué F, Steyer A, Cloetens P, Le Duc G, Fonta C. From homogeneous to fractal normal and tumorous microvascular networks in the brain. Journal of Cerebral Blood Flow \& Metabolism. 2007;27:293-303.

[7] Bravin A, Coan P, Suortti P. X-ray phase-contrast imaging: from pre-clinical applications towards clinics. Physics in Medicine \& Biology. 2012;58:R1.

[8] Momose A, Takeda T, Itai Y, Hirano K. Phase-contrast X-ray computed tomography for observing biological soft tissues. Nature medicine. 1996;2:473.

[9] Fratini M, Bukreeva I, Campi G, Brun F, Tromba G, Modregger P, et al. Simultaneous submicrometric 3D imaging of the micro-vascular network and the neuronal system in a mouse spinal cord. Scientific reports. 2015;5:8514.

[10] Maugeri L, DiNuzzo M, Moraschi M, Nicaise C, Bukreeva I, Mangini F, et al. Fractal Dimension Analysis of High-Resolution X-Ray Phase Contrast Micro-Tomography Images at Different Threshold Levels in a Mouse Spinal Cord. Condensed Matter. 2018;3:48.

[11] Canny JF. Finding edges and lines in images. MASSACHUSETTS INST OF TECH CAMBRIDGE ARTIFICIAL INTELLIGENCE LAB; 1983.

[12] Otsu N. A threshold selection method from gray-level histograms. IEEE transactions on systems, man, and cybernetics. 1979;9:62-6.

[13] Lorigo LM, Faugeras OD, Grimson WEL, Keriven R, Kikinis R, Nabavi A, et al. Curves: Curve evolution for vessel segmentation. Medical image analysis. 2001;5:195-206.

[14] Lo P, Sporring J, Ashraf H, Pedersen JJ, de Bruijne M. Vessel-guided airway tree segmentation: A voxel classification approach. Medical image analysis. 2010;14:527-38.

[15] Martínez-Pérez ME, Hughes AD, Stanton AV, Thom SA, Bharath AA, Parker KH. Retinal blood vessel segmentation by means of scale-space analysis and region growing. International Conference on Medical Image Computing and Computer-Assisted Intervention: Springer; 1999. p. 90-7.

[16] Florin C, Paragios N, Williams J. Globally optimal active contours, sequential Monte Carlo and on-line learning for vessel segmentation. European Conference on Computer Vision: Springer; 2006. p. 476-89.

[17] Lesage D, Angelini ED, Bloch I, Funka-Lea G. Medial-based Bayesian tracking for vascular segmentation: Application to coronary arteries in 3D CT angiography. 2008 5th IEEE International Symposium on Biomedical Imaging: From Nano to Macro: IEEE; 2008. p. 268-71.

[18] Schneider M, Sundar H. Automatic global vessel segmentation and catheter removal using local geometry information and vector field integration. 2010 IEEE International Symposium on Biomedical Imaging: From Nano to Macro: IEEE; 2010. p. 45-8.

[19] Zhou J, Chang S, Metaxas D, Axel L. Vascular structure segmentation and bifurcation detection. 2007 4th IEEE International Symposium on Biomedical Imaging: From Nano to Macro: IEEE; 2007. p. 872-5.

[20] Frangi AF, Niessen WJ, Vincken KL, Viergever MA. Multiscale vessel enhancement filtering. 
International conference on medical image computing and computer-assisted intervention: Springer; 1998. p. 130-7.

[21] Hernández-Vela A, Gatta C, Escalera S, Igual L, Martin-Yuste V, Radeva P. Accurate and robust fully-automatic QCA: method and numerical validation. International Conference on Medical Image Computing and Computer-Assisted Intervention: Springer; 2011.p. 496-503.

[22] Sato Y, Nakajima S, Shiraga N, Atsumi H, Yoshida S, Koller T, et al. Three-dimensional multiscale line filter for segmentation and visualization of curvilinear structures in medical images. Medical image analysis. 1998;2:143-68.

[23] Xiao C, Staring M, Wang Y, Shamonin DP, Stoel BC. Multiscale bi-Gaussian filter for adjacent curvilinear structures detection with application to vasculature images. IEEE Transactions on Image Processing. 2012;22:174-88.

[24] Benmansour F, Cohen LD. Tubular structure segmentation based on minimal path method and anisotropic enhancement. International Journal of Computer Vision. 2011;92:192-210.

[25] Law MW, Chung AC. An oriented flux symmetry based active contour model for three dimensional vessel segmentation. European conference on computer vision: Springer; 2010. p. 72034.

[26] González G, Aguet F, Fleuret F, Unser M, Fua P. Steerable features for statistical 3D dendrite detection. International Conference on Medical Image Computing and Computer-Assisted Intervention: Springer; 2009. p. 625-32.

[27] Jacob M, Unser M. Design of steerable filters for feature detection using canny-like criteria. IEEE transactions on pattern analysis and machine intelligence. 2004;26:1007-19.

[28] Schneider M, Hirsch S, Weber B, Székely G, Menze BH. Joint 3-D vessel segmentation and centerline extraction using oblique Hough forests with steerable filters. Medical image analysis. 2015;19:220-49.

[29] Stefanutti E, Sierra A, Miocchi P, Massimi L, Brun F, Maugeri L, et al. Assessment of the effects of different sample perfusion procedures on phase-contrast tomographic images of mouse spinal cord. Journal of Instrumentation. 2018;13:C03027.

[30] Mittone A, Manakov I, Broche L, Jarnias C, Coan P, Bravin A. Characterization of a sCMOSbased high-resolution imaging system. Journal of synchrotron radiation. 2017;24:1226-36.

[31] Paganin D, Mayo S, Gureyev TE, Miller PR, Wilkins SW. Simultaneous phase and amplitude extraction from a single defocused image of a homogeneous object. Journal of microscopy. 2002;206:33-40.

[32] Brun F, Massimi L, Fratini M, Dreossi D, Billé F, Accardo A, et al. SYRMEP Tomo Project: a graphical user interface for customizing CT reconstruction workflows. Advanced structural and chemical imaging. 2017;3:4.

[33] Freeman WT, Adelson EH. The design and use of steerable filters. IEEE Transactions on Pattern Analysis \& Machine Intelligence. 1991:891-906.

[34] Kumar AT, Chung E, Raymond SB, van de Water JA, Shah K, Fukumura D, et al. Feasibility of in vivo imaging of fluorescent proteins using lifetime contrast. Optics letters. 2009;34:2066-8.

[35] Schneider CA, Rasband WS, Eliceiri KW. NIH Image to ImageJ: 25 years of image analysis. Nature methods. 2012;9:671. 


\section{Table}

Table 1. Contrast-to-background ratios and filter gain. List of measured contrast-to-background ratios, and corresponding filter gain, for the images presented in this work (as labelled in the first column). The subscript 'FR' denotes the CBRs as determined on the FR maximum projected image.

\begin{tabular}{|l|l|l|l|l|}
\hline Figures & $\boldsymbol{c} \mathbf{V}, \mathbf{F R} / c_{\mathbf{V}}$ & $\boldsymbol{c}_{\mathbf{N}, \mathbf{F R} / \boldsymbol{c}_{\mathbf{N}}}$ & $\boldsymbol{c}_{\mathbf{I}, \mathbf{F R} / \boldsymbol{c}_{\mathbf{I}}}$ & Note \\
\hline $6 \mathrm{~B}, \mathrm{C}$ & $56.1 / 3.35=17$ & $\mathrm{n} / \mathrm{a}$ & $40.9 / 5.99=6.8$ & Microfil $^{\circledR}$ \\
\hline $8 \mathrm{~A}, \mathrm{~B}$ & $3.35 / 0.0572=57$ & $1.94 / 0.0839=23$ & $3.08 / 0.110=28$ & PFA perf. \\
\hline $8 \mathrm{C}, \mathrm{D}$ & $6.28 / 0.125=50$ & $\mathrm{n} / \mathrm{a}$ & $5.81 / 0.174=33$ & Ethanol perf. \\
\hline
\end{tabular}

\section{Figures}

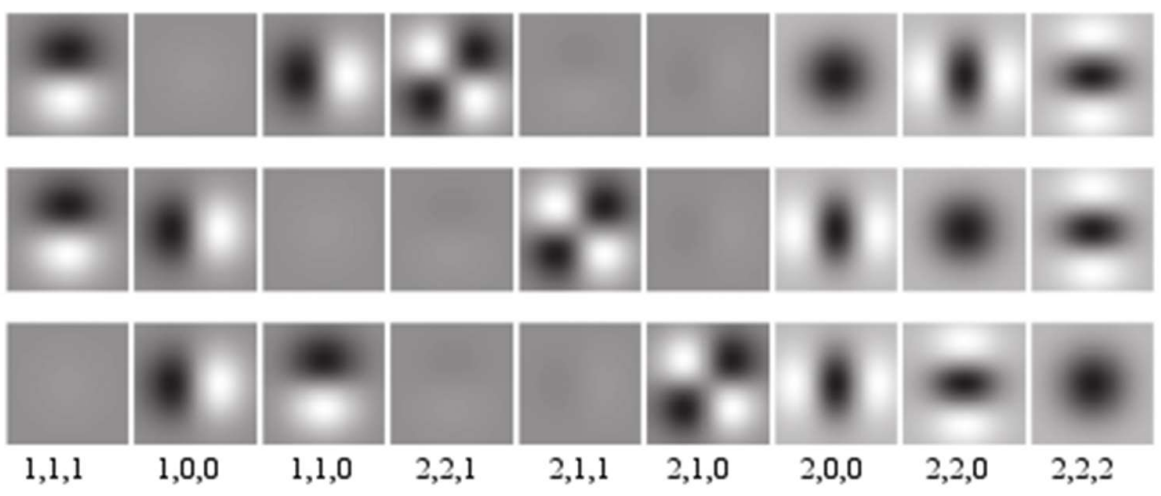

Figure 1. Filter templates. From top to bottom: sagittal, coronal and axial central cross-sections of the filter templates for various orders (as labelled at the bottom) and without rotation. The size of each cross-section is $6 \sigma$. 


\section{Filter orientation}

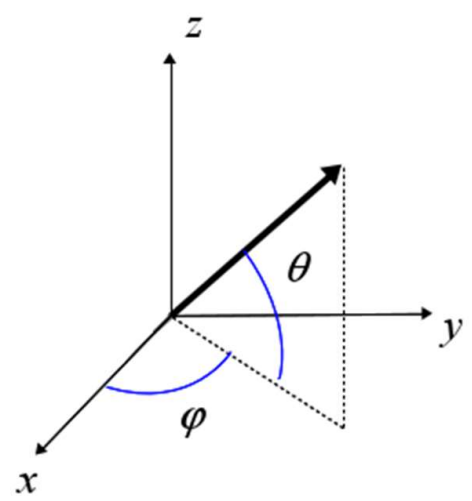

Figure 2. Filter 3D orientation. Elevation $(\theta)$ and azimuth $(\varphi)$ determine the FT direction. The $x y$ plane is parallel to each tomographic slice plane. 


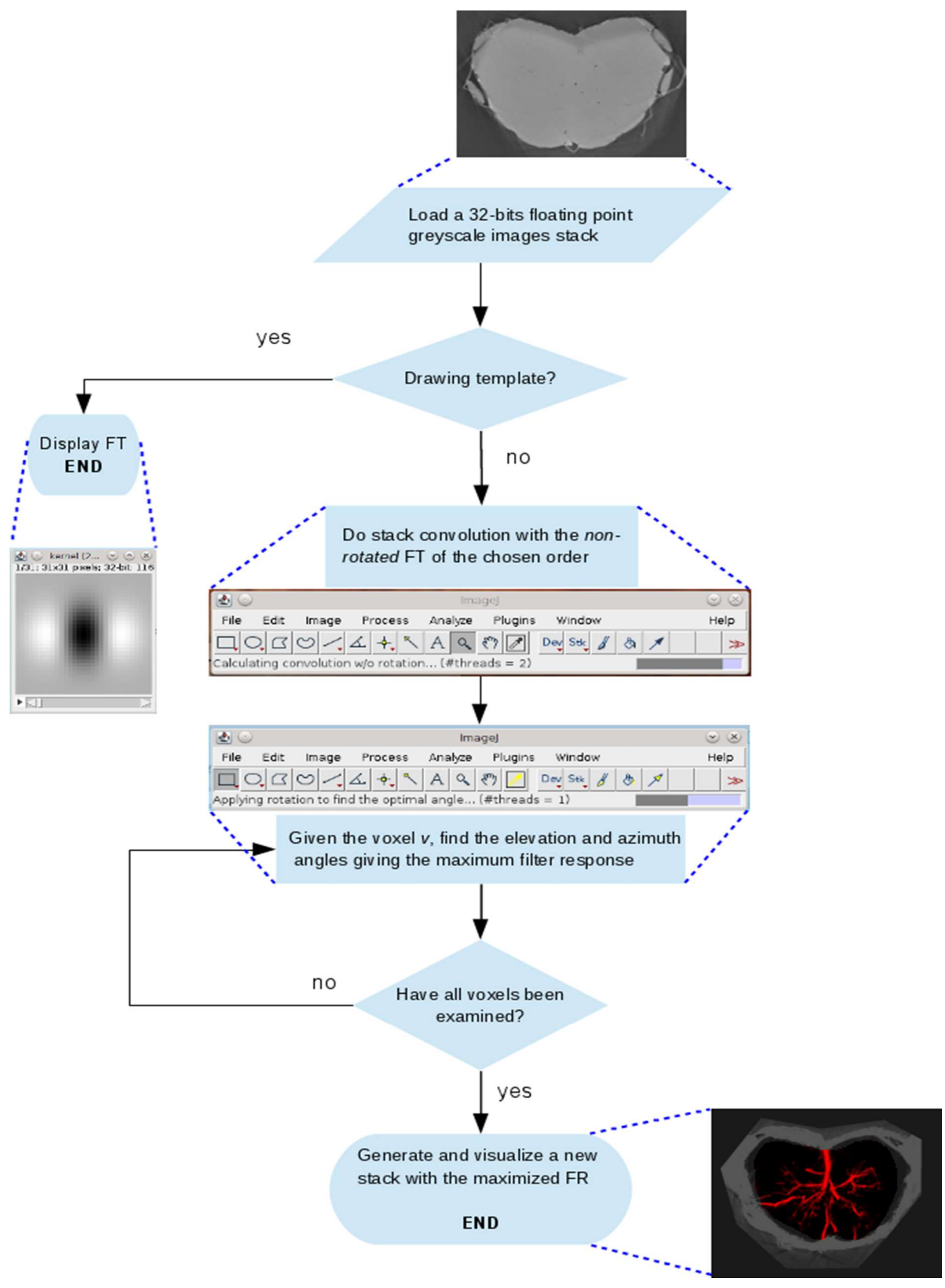

Figure 3. Steerable3D workflow. Flowchart of the plugin working steps. 


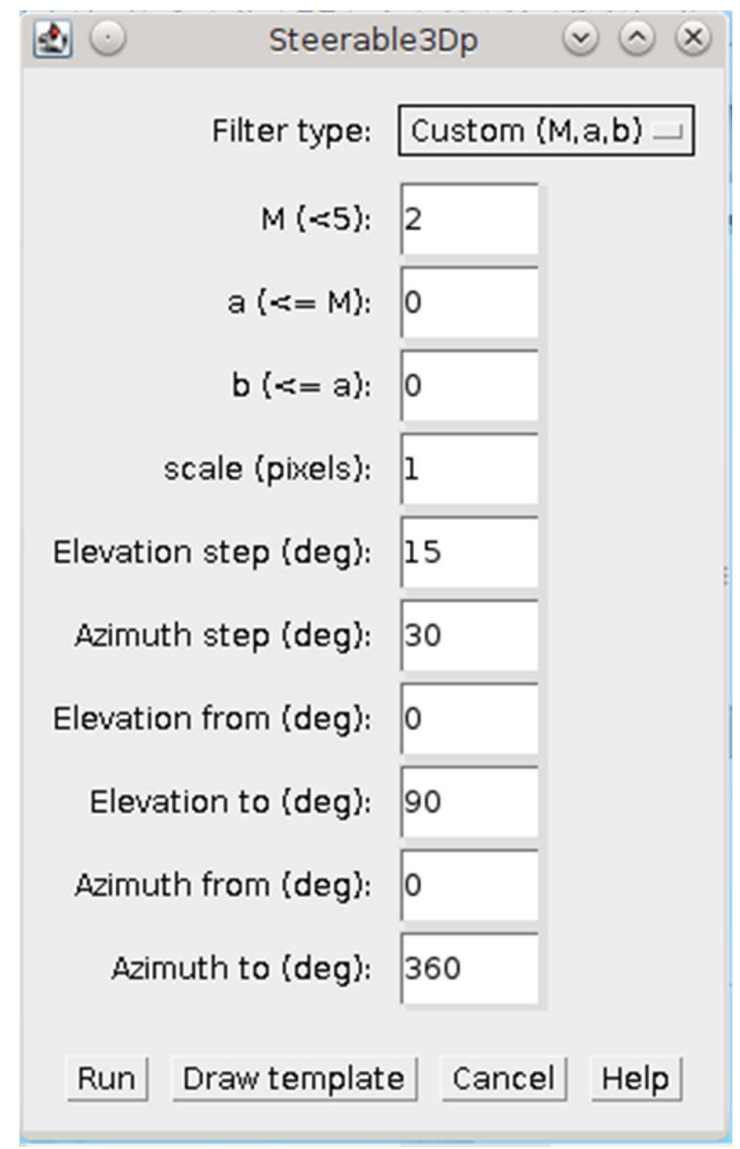

Figure 4. Plugin GUI. 'Elevation step' refers to $\Delta \theta$; 'Azimuth step': $\Delta \varphi$; 'Elevation from': $\theta_{1} ;$;Elevation to': $\theta_{2}$ 'Azimuth from': $\varphi_{1 \square}$ and 'Azimuth to': $\varphi_{2} \square$ 


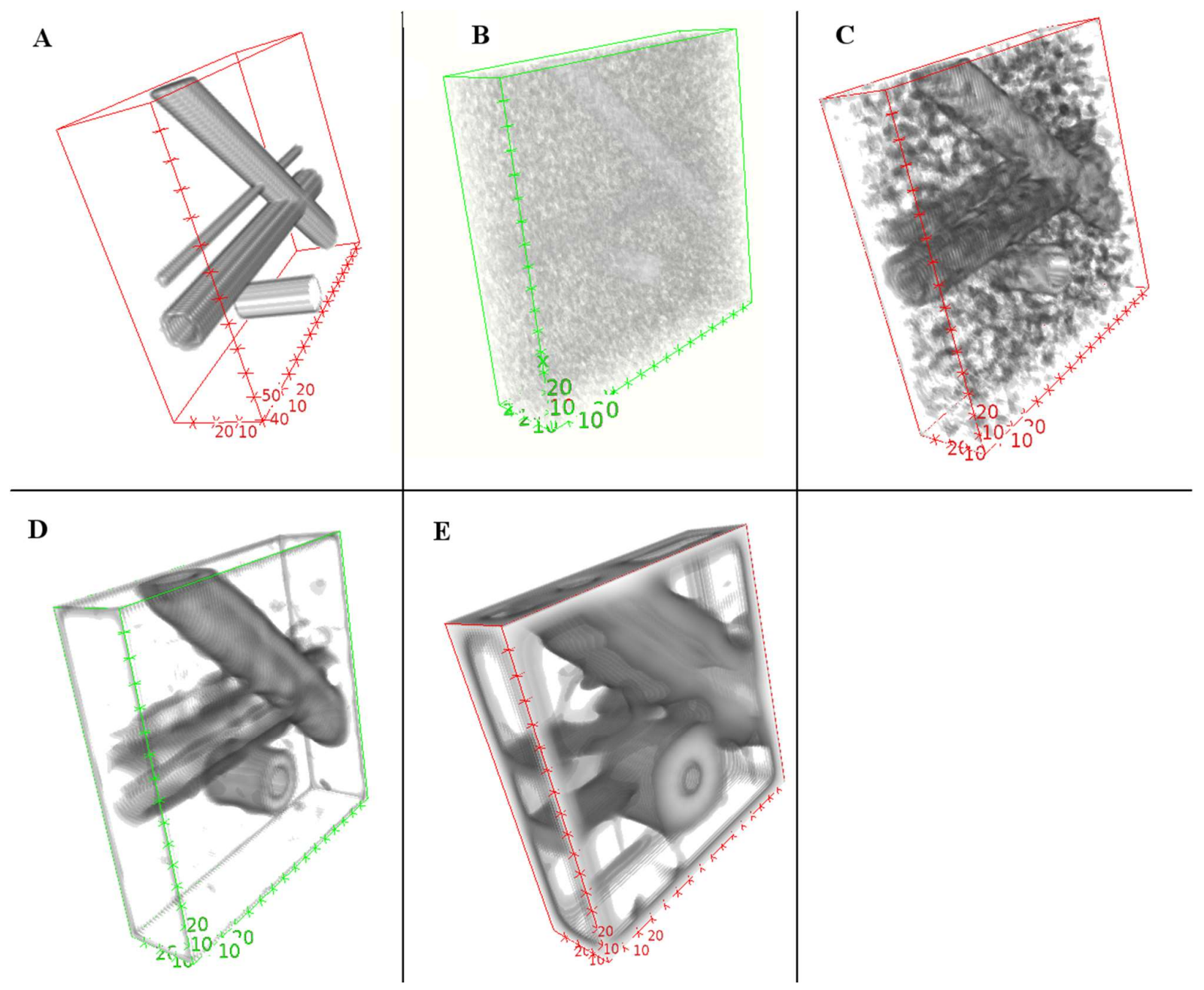

Figure 5. Filtering a synthetic image. (A) Four synthetic "vessels" generated as cylinders of various size and orientation within a box of $140 \times 140 \times 60$ pixels. They have either 8 or 2 voxels width. (B) The same as in (A) but with a white noise added with an average grey level equal to the grey level of the "vessels"; in this case a certain degree of transparency had to be set in the 3-D rendering to improve visibility. (C, D, E) FT $(2,0,0)$ responses of the (B) volumetric image using a scale $\sigma=2,4$, 8 , respectively. 


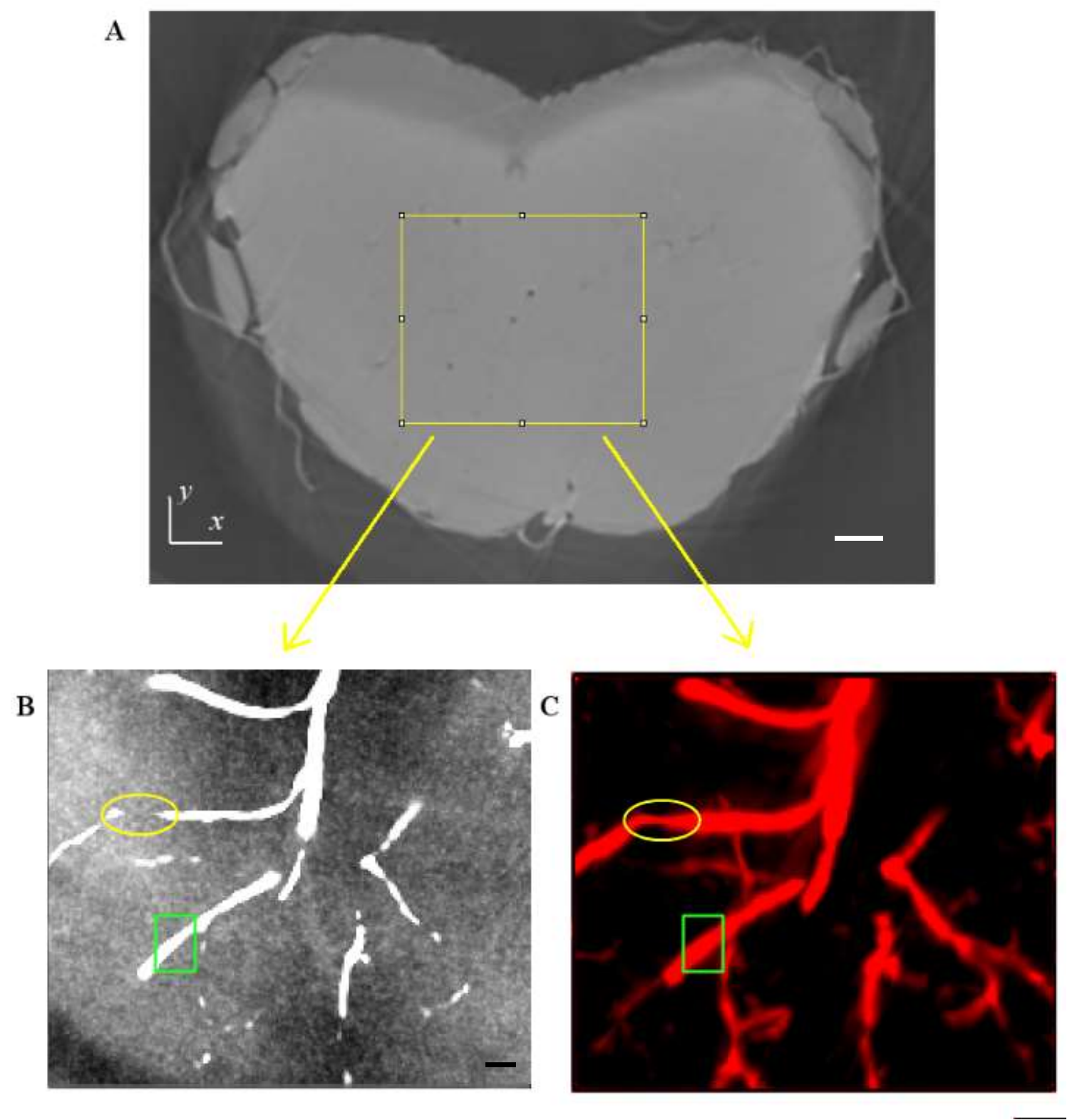

Figure 6. Filter test on a $\mathrm{SxrPC} \mu \mathrm{T}$ image of a mouse spinal cord perfused with contrast agent.

(A) Axial cross-section and a VOI with size $200 \times 170 \times 100$ voxels $(1$ voxel size $=3.5 \mu \mathrm{m})$ enclosed in a yellow contour (the white scale bar is 150 micron). (B) Projection of the VOI of (A). (C) maximum projection of the S3D response stack (FT order 2,0,0 with $\sigma=2$ ). The ramifications of the anterior (sulcal) artery are clearly visible. The green rectangles indicate the regions used to evaluate the CBR on a vessel (see text). Yellow ovals enclose a region in which a vessel discontinuity is revealed as only apparent by the FR contrast enhancement. (scale bar in B and C is 30 micron) 


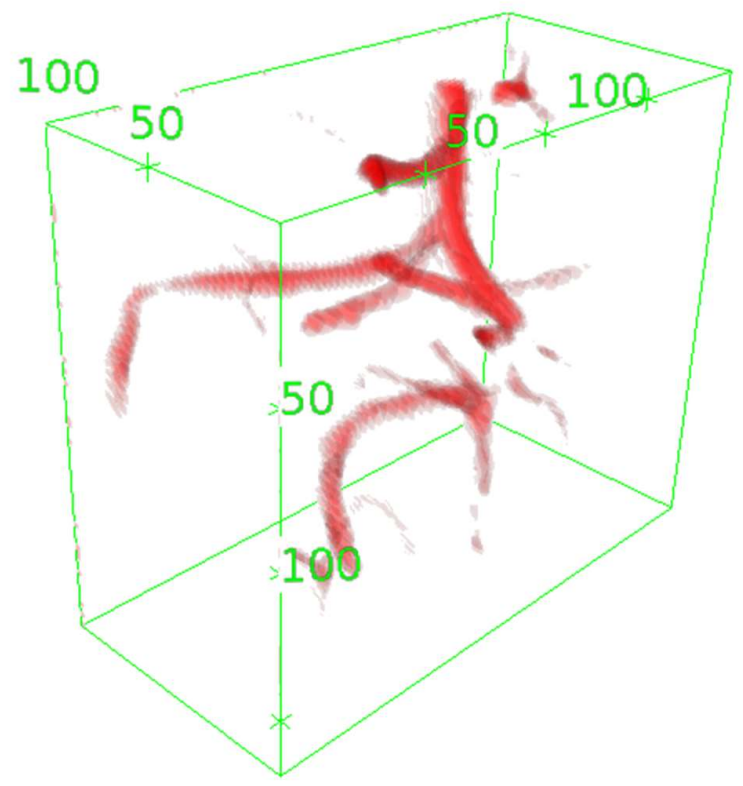

Figure 7. Filter response 3-D rendering. Three-dimensional structure of the vascular network in the volumetric FR (projected in Fig 6). 

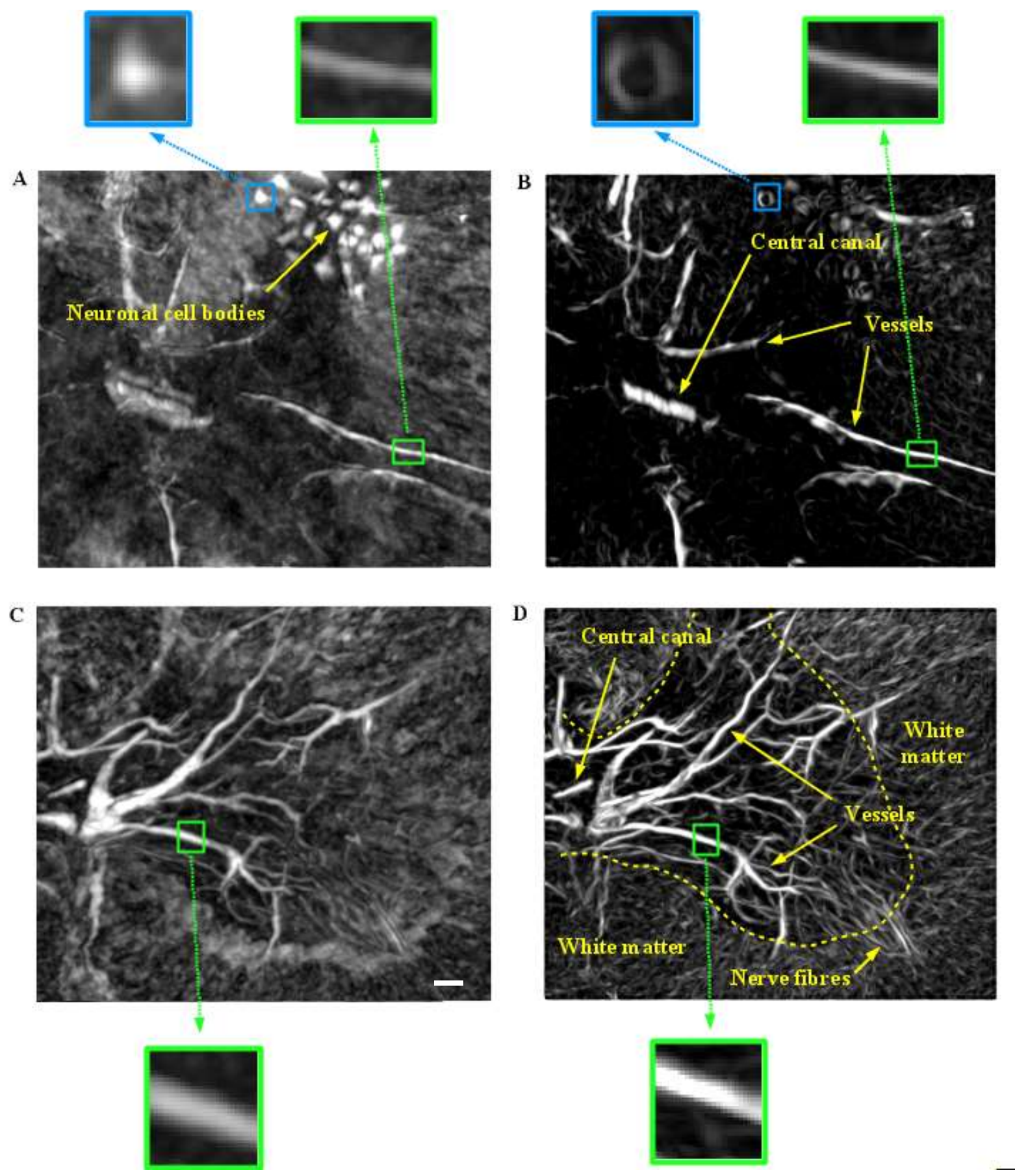

Figure 8. Filter test on SxrPC $\mu \mathrm{T}$ images of a mouse spinal cord without contrast agent. (A) Projected VOI (see text) with size $500 \times 440 \times 200$ pixels (pixel size $=1.6 \mu \mathrm{m}$ ) in the same region as in Fig. 6 of a PFA perfused spinal cord. (B) Projected maximum of the FR stack of the VOI (FT order 2,0,0 with $\sigma=2)$. (C), (D) The same as (A) and (B), respectively, but with ethanol perfusion. The yellow dashed curve roughly delimits white and grey matters. The small rectangular regions used for 
the CBR calculation on a vessel (green) and a neuron (blue) are also enlarged and reported. In (D) the dense neural fibres network appears to be resolved in the white matter region. (white scale bar 20 micron)

A

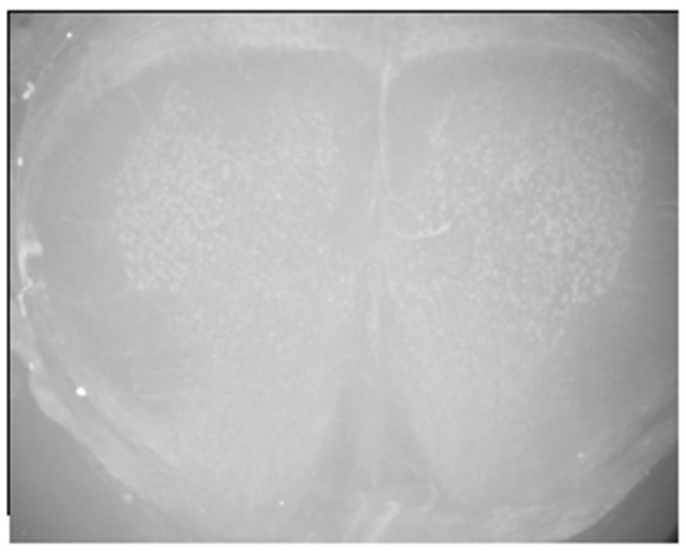

B

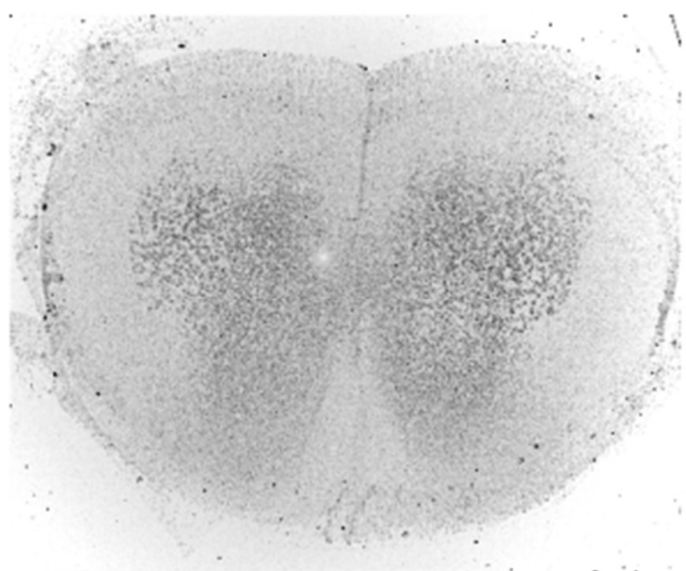

Figure 9. Filter response on grey/white matter. (A) Projected maximum of a $200 \mathrm{SxrPC} \mu \mathrm{T}$ slices stack of a mouse spinal cord. (B) Projected maximum of the S3D volumetric response of (A).

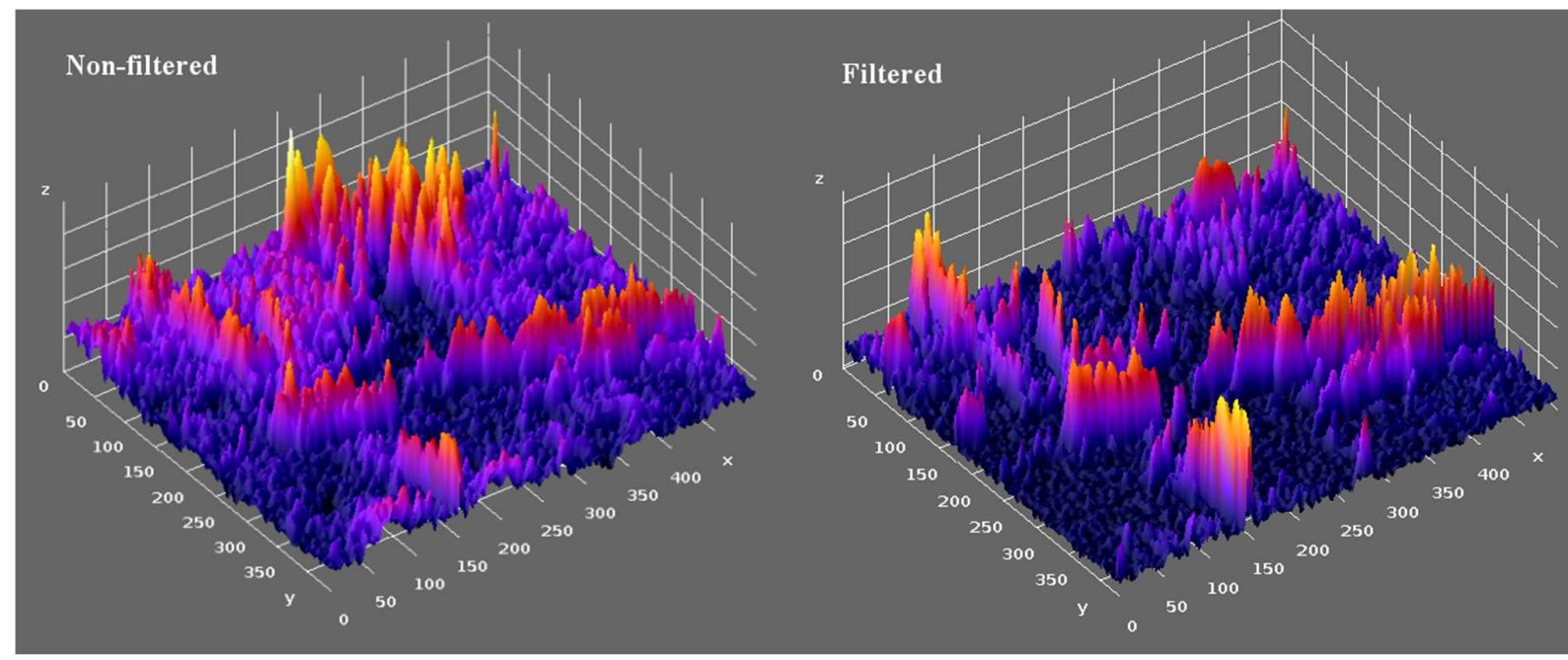

Figure 10. Grey levels surface plot of the PFA perfused case. For each pixel at $(x, y)$ in Figs $8 \mathrm{~A}$ and $8 \mathrm{~B}$, the grey level is reported on the z-axis in the non-filtered and filtered plot, respectively. 
